\title{
CONSUMPTION OF VITAMIN A RICH FOODS AND DARK ADAPTATION THRESHOLD OF PREGNANT WOMEN AT DAMOT SORE DISTRICT, WOLAYITA, SOUTHERN ETHIOPIA
}

\author{
Hiwot Abebe ${ }^{1}$, Yewelsew Abebe ${ }^{1}$, Eskindir Loha ${ }^{1}$, Barbara J. Stoecker ${ }^{2}$
}

ABSTRACT

BACKGROUND: More than 7.2 million pregnant women in developing countries suffer from vitamin $A$ deficiency. The objective of this study was to assess dark adaptation threshold of pregnant women and related socio-demographic factors in Damot Sore District, Wolayita Zone, Southern Ethiopia.

METHODS: A cross-sectional study design was employed to collect data from 104 pregnant women selected by a two stage cluster sampling. A Dietary Diversity Score was calculated by counting the number of food groups consumed by the women in 24 hour period prior to the study. Scotopic Sensitivity Tester-1 was used to test participant's pupillary response to graded amounts of light in a dark tent.

RESULTS: Half of the pregnant women in this study had dietary diversity score less than three. The majority of participants (87.5\%) had consumed either animal or plant source vitamin A rich foods less than three times a week. For a unit increase in individual dietary diversity score, there was a decrease in dark adaptation measurement by $0.29 \mathrm{log} \mathrm{cd} / \mathrm{m}^{2}(p=0.001)$. For a unit increase in gestational week of pregnancy, there was an increase in dark adaptation measurement by $0.19 \mathrm{log} \mathrm{cd} / \mathrm{m}^{2}(P=0.027)$.

CONCLUSIONS: Results from this study indicated that the pregnant women had low consumption of vitamin A rich foods, and their dark adaptation threshold increases with gestational age indicating that their vitamin A status is getting worse. There is a need to design appropriate intervention and target this group of population.

KEYWORDS: Vitamin A deficiency, pregnant women, dark adaptation threshold, Southern Ethiopia

DOI: http://dx.doi.org/10.4314/ejhs.v24i3.5

\section{INTRODUCTION}

More than 7.2 million pregnant women in developing countries suffer from vitamin A deficiency (VAD) (1). Studies indicate that VAD is still a major public health problem among Ethiopian pregnant women (2-4) with severe health consequences for both the mother and the fetus including pre-term delivery, pregnancy induced hypertension and moderate to severe anemia (5).

Ethiopian Demographic and Health Survey (EDHS) in 2011 indicated only 16\% of women that gave birth in the past five years before the survey had received postpartum vitamin A supplementation (6). Plant foods contribute to more than $80 \%$ of the vitamin A intake in African households (7). Even though the best sources of readily bioavailable vitamin A are animal source foods such as liver, eggs and dairy products (8), low income households cannot afford to consume these foods on a regular basis. As a result, high rates of deficiency in micronutrients including vitamin $\mathrm{A}$ are common among resource poor population groups with such type of dietary pattern (9).

A review on prevalence of vitamin A deficiency indicated that Ethiopia has made inadequate progress in addressing vitamin A deficiency over the last 50 years despite several

\footnotetext{
${ }^{1}$ Institute of Nutrition, Food Science and Technology, Hawassa University, Hawassa, Ethiopia

${ }^{2}$ Oklahoma State University, Stillwater, OK, USA

Corresponding Author: Hiwot A. Hailessilasie, Email:Hiwotzi@yahoo.com
} 
Attempts made to alleviate the problem (10). A recent study in Southern Ethiopia also showed that $37.9 \%$ pregnant women had VAD (3). Different techniques have been proposed for assessing vitamin A status at population level. History of night blindness during pregnancy is one of the simple methods that can be used to assess vitamin A deficiency. Dark adaptation threshold, a functional measure of vitamin A status strongly associated with serum retinol concentration $(11,12)$ can also be used to assess vitamin A status and avoid the subjective nature of history of night blindness. Depletion of vitamin A leads to early impairment of dark adaptation, which is being unable to see low light intensity (13). A study conducted in northern Ethiopia revealed that women having serum retinol values below $35 \mu \mathrm{g} / \mathrm{dl}$ had reduced sensitivity to low light stimulation. Further, in a similar study, it was found that dark adaptation was strongly associated with serum retinol concentration (14).

Assessing vitamin A status of vulnerable groups and factors associated with it is an important step for planning interventions. The objective of this study was thus to assess dark adaptation threshold of pregnant women and related socio-demographic factors in Damot Sore District, Wolayita Zone, Southern Ethiopia.

\section{MATERIALS AND METHODS}

Study Setting and Study Period: The study was conducted in Damot Sore District, one of the 13 districts in Wolayita Zone, with a total population of 112 , 909 , i.e. 624.1 people per $\mathrm{km}^{2}$ population density and $180.92 \mathrm{~km}^{2}$ total area (15). A cross-sectional survey was employed to collect data from January 1 to February 28, 2009.

Sample Size and Sampling Procedure: Single population mean formula was used to calculate the sample size. A value of a standard normal distribution score at confidence level of $95 \%$, pooled standard deviation calculated from a study on dark adaptation pattern of pregnant women (14), and desired degree of accuracy set at 0.03 was inserted in the formula and a sample size of 52 was obtained. Design effect of 2 was considered and the resulting sample size was 104 pregnant women.
The desired number of pregnant women was then selected by a two-stage cluster sampling method. The first step was selecting 3 kebeles by probability proportional to size. Sampling interval was calculated by dividing cumulative population of pregnant women by 3 , number of kebeles to be selected. Using Microsoft Excel random number generating system from numbers between 1 and the sampling interval, a random number was chosen. Then, the first kebele with the random number was selected. The next kebeles were selected by adding the sampling interval on the random number, and taking the kebele with that number. The second stage was selecting pregnant women; they were selected randomly from list of all pregnant women. The list was generated before data collection using the register in the nearby health post and also by going house to house with the help of health extension workers. Ethical approval was obtained from Ethical Committee of Hawassa University. Oral consent was obtained from all the study participants.

Dark Adaptation Threshold: Dark adaptation threshold of pregnant women was assessed by Scotopic Sensitivity Tester-1 (LKC Technologies, Inc, Gaithersburg, MD). Tent as described by Sanchez and colleagues (16) was constructed at a health post in each kebele to create a dark room for the test. Blankets were pinned along the bottom of the tent to prevent light entering the tent, and black masking tape was used to cover holes as needed. Inability of a fully dark adapted observer to read black letters $1.5 \mathrm{~cm}$ thick on a white background was used to check the appropriateness of darkness in all tests. Dark adaptation was tested using modified technique descried elsewhere (17). SST-1, which consists of a hand-held stimulator and a control unit, was used to test women's pupillary response to graded amounts of illumination. The light presented by the instrument ranged from 0 to 30 decibel intensities. Prior to dark adaptation, each participant was subjected to bleaching at a distance of 1 meter with a digital camera flash. Six participants were dark adapted together in the tent for 10 minutes and then tested individually. The women were asked to focus at a distance of 2 meter. The hand held stimulator was placed against each participant's 
left eye and the intensity of light was increased until the pupil in the right eye contracted in two consecutive trials. Pupillary response of the pregnant women in this study was evaluated by use of a night vision scope (ELF-1, LOMO America Inc.). Intensity of the light that caused contraction was recorded and converted to $\log$ $\mathrm{cd} / \mathrm{m}^{2}$, which is SI unit of luminance using values set during calibration.

Dietary Diversity: Dietary diversity score (DDS) could serve as a proxy of nutrient adequacy of an individual's diet; strong association was found between diet diversity and micronutrient adequacy in adult women (19). In this study, DDS was calculated using a questionnaire adopted from Food and Agriculture Organization (FAO) guidelines (18). Women involved in the study were asked separately to recall all the dishes, snacks, or other foods eaten in the previous 24 hours prior to the survey, regardless of whether the food was eaten inside or outside the house. During the data collection, each woman was prompted to make sure that no meal or snack was forgotten. Next, detailed list of all the ingredients of the dishes, snacks, or other foods mentioned in the reported consumed food were collected from each woman via interview. Food frequency questions with lists of locally available vitamin A rich foods identified prior the survey were used. The study participants were then asked to recall how many days they consumed each of the 15 locally available vitamin A rich foods in the past seven days. During training of data collectors, it was stressed that small quantities of food eaten less than 1 tablespoon should be excluded. This was important as foods eaten below the aforementioned quantities would not contribute significantly to nutrient adequacy but would inflate the score. Minimum consumption of 1 tablespoon of food counted in DDS was better correlated with probability of adequacy (19). Food items that belong to more than one food group such as pepper that could be assigned as either vegetable or spice were decided in advance to avoid double counting.

Wealth Index: Household assets (radio, tape, television, bicycle, hand torch, horse or donkey cart), housing conditions (roof material, number of rooms, wall type, windows, availability and type of latrine), land size in hectare, and ownership of domestic animals were included in the wealth index according to the method described by Gwatkin and colleagues (20). Each asset was assigned a score between 0 and 1 where an increased value reflected better status. These scores were then summed to get a single wealth index. The study participants were ranked according to the wealth index score and divided into quintiles, from the lowest (first quintile) to the highest (fifth quintile).

Data quality: Before the actual data collection, the SST-1 and the questionnaire were pre tested on ten pregnant women. Seven health promoters who completed at least high school were selected and trained to collect data using the questionnaire. Dark adaptation threshold assessment was conducted by the researcher. The intra observer reliability of the researcher was checked until it became within a difference of 1 unit score for 10 successive subjects.

Data Analysis: SPSS (v. 16) software package was used to analyze the data. Mean \pm standard deviations were reported and $p$ values less than 0.05 were considered as statistically significant. Normality assumption was checked using Kolmogorov-Smirnov test before further analysis. An independent sample t-test was used to compare mean differences between two groups, whereas ANOVA test was used to compare mean differences for more than two groups. Least significant difference testing was then performed in the case of ANOVA to determine which group differed significantly. Linear regression analysis was used to determine variables that predicted the dark adaptation threshold.

\section{RESULTS}

The mean $( \pm S D)$ age of study population was $27.5 \pm 6.1$ years. The mean gestational week of the pregnant women involved in the study was $28 \pm 7$ weeks, and $20(19.2 \%)$ were primiparas. Eighty four women $(80.7 \%)$ had been pregnant at least once. Selected socio-demographic characteristics of the study participants are shown in Table 1. 
Table 1: Socio-demographic characteristics of study participants in Damot Sore $(\mathrm{N}=104)$

\begin{tabular}{lll}
\hline $\begin{array}{l}\text { Socio-demographic } \\
\text { variables }\end{array}$ & Frequency & Percent \\
\hline $\begin{array}{ll}\text { Age group (in years) } \\
\text { 15-20 }\end{array}$ & 13 & 12.5 \\
$20-24$ & 27 & 26.0 \\
$25-29$ & 19 & 18.3 \\
$30-34$ & 33 & 31.7 \\
$\quad 35-49$ & 12 & 11.5 \\
Education & & \\
$\quad$ No formal education & 61 & 58.7 \\
$\quad$ Read and write only & 32 & 2.9 \\
$\quad$ Primary education & 8 & 30.8 \\
$\quad$ Secondary education or & 3 & 7.7 \\
$\quad$ college & & \\
Occupation & & \\
$\quad$ House wife & 85 & 81.7 \\
$\quad$ Local trader & 17 & 16.3 \\
$\quad \begin{array}{l}\text { Other Employment } \\
\text { Family size }\end{array}$ & 2 & 1.9 \\
2-4 family member & 44 & 42.3 \\
5-7 family member & 49 & 47.1 \\
$\quad$ 7 family member & 11 & 10.6 \\
\hline
\end{tabular}

The food items consumed by the study participants in the 24 hours before the survey are presented in Table 2. Dietary diversity score of the study participants showed that half $(50 \%)$ of the pregnant women had dietary diversity scores less than three, and only 19 (18.3\%) had scores greater than six. Almost all women (97.1\%) reported consumption of cereals followed by white tubers $(82.7 \%)$ (yam, sweet potato, and potato) in the previous 24 hour before the survey (Table 2). Only $21(20 \%)$ of the pregnant women in the study reported consumption of special foods during pregnancy, which is a diet different from what they used to eat before pregnancy. Only $9(10.7 \%)$ of the eligible women received postpartum vitamin A supplementation.

Table 2: Reported food groups consumed in the previous 24 hours by pregnant women in Damot Sore $(\mathrm{N}=104)$

\begin{tabular}{lll}
\hline Food items & Frequency & Percentage \\
\hline Cereals & 101 & 97.1 \\
White tubers & 86 & 82.7 \\
Legumes & 49 & 47.1 \\
Oil and butter & 43 & 41.3 \\
Dark green leafy vegetables & 33 & 31.7 \\
Milk and milk products & 20 & 19.2 \\
Vitamin A rich fruits & 8 & 7.7 \\
Other fruits & 7 & 6.7 \\
Vitamin A rich vegetables and roots & 6 & 5.8 \\
Egg & 4 & 3.8 \\
Meat & 3 & 2.9 \\
\hline Organ meat & 2 & 1.9 \\
Other vegetables & 1 & 1.0 \\
Fish & 0 & 0 \\
\hline
\end{tabular}

From the locally available plant foods which were good sources of carotene; kale (Brassica oleracea) and pumpkin were consumed at least once a week by $72(69.2 \%)$ and $53(51 \%)$ of the pregnant women respectively, while milk was consumed at least once a week from animal source vitamin A rich foods by $89(85.6 \%)$ of the women (Table 3 ).
However, majority of the participants $(87.5 \%)$ had consumed vitamin A rich foods from animals or plants less than three times a week. Only $31(29.8 \%)$ of the women reported having heard about vitamin A mainly from health professionals $(41.9 \%)$ or neighbors $(31.2 \%)$. 
Table 3: Reported consumption of vitamin A rich foods in the previous one week before the survey, Damot Sore $(\mathrm{N}=104)$

\begin{tabular}{clllllllll}
\hline Food items & \multicolumn{7}{l}{ Frequency of intake (number of days/week) } & & \\
\cline { 2 - 9 } & Never & 1 & 2 & 3 & 4 & 5 & 6 & 7 \\
\hline Plant source \% & & & & & & & & \\
Tomato & 95.2 & 2.9 & 1 & 1 & 0 & 0 & 0 & 0 \\
& & & & & & & & \\
Carrot & 91.3 & 1.9 & 4.8 & 1.9 & 0 & 0 & 0 & 0 \\
Guava & 88.5 & 5.8 & 2.9 & 1.8 & 1.0 & 0 & 0 & 0 \\
Mango & 84.6 & 7.7 & 4.8 & 1.0 & 2.9 & 0 & 0 & 0 \\
Papaya & 68.3 & 19.2 & 6.7 & 5.8 & 0 & 0 & 0 & 0 \\
Pepper & 66.3 & 9.6 & 9.6 & 7.7 & 4.8 & 1.0 & 1.0 & 0 \\
Pumpkin & 49.0 & 21.2 & 14.4 & 7.7 & 3.8 & 1.0 & 1.0 & 1.9 \\
Kale & 31.7 & 25.0 & 27.9 & 12.5 & 2.9 & 0 & 0 & 0 \\
Animal source \% & & & & & & & & \\
Liver & 84.6 & 9.6 & 4.8 & 1.0 & 0 & 0 & 0 & 0 \\
Egg & 74.0 & 14.4 & 7.7 & 1.9 & 1.0 & 1.0 & 0 & 0 \\
Milk & 15.4 & 70.2 & 10.4 & 4 & 0 & 0 & 0 & 0 \\
\hline
\end{tabular}

The majority $(91.3 \%)$ of the participants knew a local word for night blindness. Eight (12.9\%) of 62 pregnant women who had given birth in the past three years reported experiencing night blindness during pregnancy. When two women who reported difficulty seeing in the day time were excluded, the adjusted night blindness became $10 \%$. Women from the older age (30-34 years) group and women with no formal education and those in their third trimester of pregnancy had the highest proportion of night blindness during their previous pregnancy.
Mean frequency of consumption (days/week) of vitamin A rich foods across the wealth quintiles was compared by using ANOVA (Table 4). There was a significant difference in the mean frequency of consumption of vitamin A rich animal source foods $(p=0.003)$. Consumption of plant source foods showed only marginal difference based on wealth categories ( $p=0.082$ ). The mean frequency of consumption of Vitamin A rich foods was significantly higher in women with formal education compared to women with no education (Table 4).

Table 4: Comparison of mean frequency of consumption of vitamin A rich foods (days/week) in the five wealth quintiles of study participants, Damot Sore (N=104)

\begin{tabular}{|c|c|c|c|c|c|c|}
\hline \multirow[b]{3}{*}{ Variables } & \multicolumn{6}{|c|}{ Mean frequency of intake days per week } \\
\hline & \multicolumn{6}{|c|}{ Wealth quintiles } \\
\hline & First (lowest) & Second & Third & Fourth & $\begin{array}{l}\text { Fifth } \\
\text { (highest) }\end{array}$ & p-value \\
\hline Animal source foods & $1.56 \pm 0.36^{\mathrm{b}}$ & $2.5 \pm 0.43^{b}$ & $3.0 \pm 0.41^{* a}$ & $3.10 \pm 0.43^{\mathrm{a}}$ & $3.64 \pm 0.60$ & 0.003 \\
\hline \multirow[t]{3}{*}{ Plant source foods } & $1.96 \pm 0.37$ & $3.18 \pm 0.68$ & $3.78 \pm 0.52$ & $2.77 \pm 0.37$ & $3.28 \pm 0.52$ & 0.082 \\
\hline & \multicolumn{6}{|c|}{ Education } \\
\hline & \multicolumn{3}{|c|}{ No formal education } & \multicolumn{2}{|c|}{ Formal education } & p-value \\
\hline Animal source foods & \multicolumn{3}{|c|}{$2.47 \pm 0.27$} & \multicolumn{2}{|c|}{$3.74 \pm 0.41$} & 0.011 \\
\hline Plant source foods & \multicolumn{3}{|l|}{$2.57 \pm 0.25$} & \multicolumn{2}{|l|}{$3.5 \pm 0.38$} & 0.047 \\
\hline
\end{tabular}


An independent sample t-test was used to compare the participants' dark adaptation threshold between women who received $\left(-3.36 \log \mathrm{cd} / \mathrm{m}^{2}\right)$ and those that did not receive $\left(-3.41 \mathrm{log} \mathrm{cd} / \mathrm{m}^{2}\right)$ postpartum vitamin A supplement after their last delivery $(\mathrm{p}$ value $=0.13)$. The mean dark adaptation $\left(-3.44 \log \mathrm{cd} / \mathrm{m}^{2}\right)$ for women in their third trimester was significantly worse than the one for women in second trimester (-3.73 log $\left.\mathrm{cd} / \mathrm{m}^{2}\right)(\mathrm{p}$ value $=0.001)$.
The dark adaptation threshold of pregnant woman was not significantly different across the five wealth quintiles (ANOVA, $p$ value $=0.15$ ). Likewise, the frequency of consumption of plant and animal source foods rich in vitamin A was not significantly associated with the women's dark adaptation threshold (linear regression, $\mathrm{P}$ value $=0.26$, Table 5).

Table 5: Regression analysis of variables with dark adaptation threshold ( $N=104)$

\begin{tabular}{|c|c|c|}
\hline Variables & $\mathrm{P}$ value & $\mathrm{B}[95 \% \mathrm{CI}]$ \\
\hline Individual dietary diversity score & 0.000 & $-0.29 *[-0.75,-0.1]$ \\
\hline Frequency of vitamin A rich foods & 0.082 & \\
\hline Trimester of pregnancy & 0.027 & $0.19 *[0.04,0.95]$ \\
\hline Vitamin A supplementation & 0.063 & \\
\hline
\end{tabular}

$* \mathrm{R}$ square of the model was 0.26

\section{DISCUSSION}

Ten percent of the study participants reported having night blindness during previous pregnancy in the past three years which is greater than the cut off value $(5 \%)$ set by IVACG for vitamin A deficiency to be considered a public health significance problem in the community. This was not surprising as the majority of participants $(87.5 \%)$ had consumed Vitamin A foods obtained from animals or plants less than three times a week. DDS also indicates that the study participants consumed less diversified diet.

The prevalence of night blindness $(10 \%)$ in this study was higher than the reported national $(6 \%)$ and regional night blindness (2.6\%). This difference could be because all women who gave birth in the past five years were included in calculating night blindness in EDHS, whereas only women who gave birth in the past three years were considered in the current study. The proportion of night blind women who gave birth in the past five years was calculated again for comparison purpose. The percentage of night blind women was reduced to $7.1 \%$ which is comparable to the national percentage of night blindness (6\%). However, a study conducted in a rural community of Ethiopia reported 20\% prevalence of night blindness (21), which is twice higher than the night blindness reported in this study. This higher proportion of night blindness could be due to the fact that only $21.3 \%$ of women in our study had greater than 4 children. Risk of night blindness had been reported to increase with parity greater than three (22).

Pregnant women who were in the second trimester had better mean dark adaptation threshold $\left(-3.73 \log \mathrm{cd} / \mathrm{m}^{2}\right)$ than women in the third trimester $\left(-3.44 \log \mathrm{cd} / \mathrm{m}^{2}\right)$. The risk of having difficulty seeing in the dark would increase later in pregnancy as the fetal demand for vitamin A increases (17). Linear regression analysis revealed a significant association of dark adaptation threshold with trimester of pregnancy and individual dietary diversity score. When both variables were entered into the model, the association between individual dietary diversity score, trimester of pregnancy and dark adaptation threshold remained significant. The coefficient of determination $\left(\mathrm{R}^{2}\right)$ of the model was 0.26 .

For a unit increase in individual dietary diversity score of the study participants, there was a decrease in dark adaptation measurement by $0.29 \log \mathrm{cd} / \mathrm{m}^{2} \quad(\mathrm{p}=0.001)$, indicating better vitamin A status with an improved individual dietary diversity score. Study had reported that DDS is well correlated with serum retinol level and could predict vitamin A deficiency (23). 
For a unit increase in gestational trimester of pregnancy, there was an increase in dark adaptation measurement by $0.19 \log \mathrm{cd} / \mathrm{m}^{2}$ $(\mathrm{p}=0.027)$. A study conducted in Nepal found dark adaptation thresholds to be significantly higher in pregnant women during the second and third trimesters of pregnancy than during the first trimester, indicating that pregnant women in the third and second trimester have lower vitamin A status (17).

The frequency of consumption of vitamin A rich foods was not significantly associated with the women's dark adaptation threshold. Factors like low fat consumption and plant matrix could reduce absorption of vitamin $\mathrm{A}$ in the human body (24) and consumption of vitamin A rich foods is affected by season (25). However, these were not addressed in this study and may have impacted on the association. The possible explanation for dark adaptation threshold of pregnant woman which was not significantly different across the five wealth quintiles could be linked with the fact that the main sources of vitamin A for the study participants were plant foods. The frequency of consumption of vitamin A rich plant foods did not differ by wealth. Other factors that affect vitamin A status such as disease (26) may have also contributed.

Vitamin A deficiency has public health importance in the community. Results from this study indicated that the study participants had low consumption of vitamin A rich foods which calls for food based approaches to alleviate the problem in pregnant women. The participants' dark adaptation threshold increases with gestational age indicating their vitamin A status gets worse as the pregnancy progress. Thus, there is a need to design appropriate intervention and target this group of population.

\section{ACKNOWLEDGMENTS}

We would like to thank the pregnant women who devoted their time to participate and Damot Soro District Health Office staffs, and Health Extension Workers for their unreserved support. This research was sponsored by Canada International Development (CIDA/UPCD) Project, Hawassa University.

\section{REFERENCES}

1. West KP Jr. Extent of vitamin A deficiency among preschool children and women of reproductive age. J Nutr 2002; 132: 2857S$286 \mathrm{~S}$.

2. Gebremedhin SG, Enquselassie FG, Umeta MD. Prevalence and Correlates of Prenatal Vitamin A Deficiency in Rural Sidama, Southern Ethiopia. J Health POPUL NUTR 2013; 31 (2):185-94.

3. Wondmikun Y. Lipid-soluble antioxidants status and some of its socio-economic determinants among pregnant Ethiopians at the third trimester. Public Health Nutr 2005; 8(6), 582-587.

4. Mulu A, Kassu A, Huruy K, Tegene B, Yitayaw G, Nakamori M, et al. Vitamin A deficiency during pregnancy of HIV infected and non-infected women in tropical settings of Northwest Ethiopia. BMC Public Health 2011; 11:569-576.

5. Radhika MS, Bhaskaram P, Balakrishna N, Ramalakshmi BA, Devi S, Kumar BS. Effects of vitamin A deficiency during pregnancy on maternal and child health. BJOG 2002; 109:689-693.

6. Central Statistical Agency [Ethiopia] and ICF International. Ethiopia Demographic and Health Survey 2011. Addis Ababa, Ethiopia and Calverton, Maryland, USA: Central Statistical Agency and ICF International. 2012.

7. Codjia G. Food sources of vitamin A and provitaminA specific to Africa: FAO perspective. Food Nutr Bull 2001; 22(4): 913.

8. WHO (World Health Organization) and Food and Agricultural Organization of the United Nations (FAO). Guidelines on food fortification with micronutrients. 2006; 1341.

9. Hess SY, Thurnham DI, Hurrell RF. Influence of provitaminA carotenoids on iron, zinc, and vitamin A status. Harvestplus Technical Monograph Series 6. Washington, DC and Cali: International Food Policy Research Institute (IFPRI) and International Center for Tropical Agriculture (CIAT). 2005; 1-28. 
10. Haidar J, Demissie T, Kloos H. Vitamin A deficiency in Ethiopia in the last 50 years. Sight and Life Magazine. 2008; 25-31.

11. Russell RM. The vitamin A spectrum: from deficiency to toxicity. Am J Clin Nutr 2000; 71: 878-884.

12. Congdon N, West KP Jr. Physiologic indicators of Vitamin A Status. In: Proceedings of the $\mathrm{XX}$ International Vitamin A Consultative Group Meeting. $J$ Nutr 2002; 132: 2889S-2894S.

13. Wasantwisut E. Recommendations for monitoring and evaluating vitamin A programs: outcome indicators. In: Proceedings of the XX International Vitamin A Consultative Group Meeting. $J$ Nutr 2002; 132(9): 2940S-2942S.

14. Wondmikun Y. Dark adaptation pattern of pregnant women as an indicator of functional disturbance at acceptable serum vitamin A levels. Eur J Clin Nutr 2002; 56(5): 462-466.

15. CSA (2009) National Statistical Abstracts, http://www.csa.gov.et/index.php/2013-0220-13-43-35/national-statisticsabstract/141-population, accessed on October, 18, 2013

16. Sanchez, A, Congdon, N, Sommer, A, et al. Pupillary threshold as an index of population vitamin A status among children in India. Am J Clin Nutr 1997; 65: 61-66.

17. Congdon N, Dreyfuss ML, Christian P, et al. Responsiveness of dark-adaptation threshold to vitamin A and beta-carotene supplementation in pregnant and lactating women in Nepal. Am J Clin Nutr 2000; 72(4): 1004-1009.

18. Food and Agriculture Organization (FAO). Guidelines for Measuring Household and Individual Dietary Diversity, version 2, Rome, Italy. 2007; 1-20.

19. Arimond M, Wiesmann D, Becquey E, et al. Simple food group diversity indicators predict micronutrient adequacy of women's diets in 5 diverse, resource-poor settings. $J$ Nutr 2010; 140:S2059-69.

20. Gwatkin DR, Rutstein, S, Johnson K, Suliman E, Wagstaff A, and Amouzou A. Socio-Economic Differences in Health, Nutrition, and Population: Ethiopia, Country Reports on HNP and Poverty, Washington, D.C. World Bank, 2007. http://siteresources.worldbank.org/INTPA H/Resources/4003781178119743396/ethio pia.pdf accessed on April, 2010

21. Umeta M, West CE, Verhoef H, Haidar J, Hautvast JG. Factors associated with stunting in infants aged 5-11 months in the Dodota-Sire District, rural Ethiopia. J Nutr 2003; 133: 1064-1069.

22. Semba RD, De Pee S, Panagides D, Poly $\mathrm{O}$, Bloem MW. Risk factors for night blindness among women of childbearing age in Cambodia. Eur J Clin Nutr 2003; 57: 1627-1632.

23. Fujita, M., Lo, Y J. and Baranski, J. R. Dietary diversity score is a useful indicator of vitamin A status of adult women in Northern Kenya. Am J Hum Biol 2012; 24: 829-834.

24. West CE, Castenmiller JJ. Quantification of the "SLAMENGHI" factors for carotenoid bioavailability and bioconversion. Int $J$ Vit Nutr Res 1998; 68: 371-377.

25. Faber M, Laubscher R. Seasonal availability and dietary intake of bcarotene-rich vegetables and fruit of 2year-old to 5-year-old children in a rural South African setting growing these crops at household level. Int J Food Sci Nutr 2008; 59, 46-60.

26. Demissie T, Ali A, Mekonnen Y, Haider J, Umeta M. Demographic and health-related risk factors of subclinical vitamin A deficiency in Ethiopia. J Health Popul Nutr 2009; 27:666-73. 